\title{
Dynamic changes and effects of environmental tobacco smoke exposure and weaning time on gut microbiota in young children aged $0-2$ years
}

\section{Tianqu Xie}

Wuhan University https://orcid.org/0000-0003-3681-9707

Yanqun Liu ( $D$ liuyanqun1984@163.com )

Wuhan University https://orcid.org/0000-0003-3586-299X

Xiaoli Chen (D846730310@qq.com)

Wuhan University

Jing He

Wuhan University

Yun Yu

Wuhan University

Yuchen Wang

Wuhan University

Jinbing Bai

Emory University

\section{Research}

Keywords:

Posted Date: March 10th, 2020

DOI: https://doi.org/10.21203/rs.3.rs-16591/v1

License: (c) (1) This work is licensed under a Creative Commons Attribution 4.0 International License.

Read Full License 


\section{Dynamic changes and effects of environmental tobacco smoke exposure and weaning time on gut microbiota in young children aged 0-2 years}

This is a cohort study.

Authors:

Tianqu Xie (2015302280014@whu.edu.cn), Yanqu Liu* (Tel: 027-68758591, E-mail: liuyanqun1984@163.com) and Xiaoli Chen*(Tel: 027-68758591, E-mail:

chenx172@whu.edu.cn), Jin He(jing26@whu.edu.cn), Yun Yu(yuyun7169@163.com), Yuchen Wang(wang-yuchen@whu.edu.cn), : Affiliation Wuhan University School of Health Sciences, Wuhan University, 169 Donghu Road, Wuhan 430071, China; Jinbing Bai(jinbing.bai@emory.edu): Affiliation Emory University Nell Hodgson Woodruff School of Nursing, 1520 Clifton Road, Atlanta, GA 30322, USA;

* means corresponding authors. 


\section{Abstract}

(1) Background: The colonization characteristics of infant gut microbiota are influenced by many factors at various stages, but few studies have explored the longitudinal effects of environmental tobacco exposure and quantitative weaning time on young children' intestinal flora. The purpose of this study was to characterize the evolution of intestinal microflora in young children aged 0-2 years and the longitudinal effects of environmental tobacco exposure and weaning time on young children aged 6 , 12, and 24 months. (2) Methods: A total of 37 maternal and children pairs were included in a tertiary general hospital in China and followed up for 2 years. General demographic information was collected on mothers (after delivery, 6 months, 12 months) and young children (6 months, 12 months, 24 months), including frequency of exposure and time of weaning, through self-made questionnaires. Fecal samples were collected from mothers in the third trimester of pregnancy and infants at 6, 12 and 24 months, and analyze the microbiota results using the V3-V4 gene sequence of 16S rRNA. (3) Results: the diversity of intestinal microflora in young children was the highest at 24 months and most similar to that in mothers. Weaning time was positively correlated with Lactobacillus in the intestines of infants aged 0 to 12 months. The diversity of microbiota exposed to environmental tobacco smoke at 6 months was lower than that of the non-exposed group, and the higher the exposure at 6-12 months, the lower the abundance of Blautia. (4) Conclusion: The gut microbiota of young children becomes more mature and complicated with age. The extension of complete weaning time to about 12 months is conducive to the colonization of beneficial bacteria. And the long-term exposure of children and the environment of tobacco smoke will affect the dysbiosis of gut microbiota. 


\section{Introduction}

The human gut contains approximately $1 \times 10^{14}$ bacterial cells, which have $>100$ times the number of genes of the human genome ${ }^{[1]}$. The gut microbiota affects the host in multiple ways, altering the growth and development of the human body and immune metabolism. Gut microbiome dysbiosis is associated with many diseases, such as type 1 diabetes in adolescent ${ }^{[2]}$, autism ${ }^{[3]}$, obesity ${ }^{[4]}$ and allergic diseases ${ }^{[5]}$.

Microorganism colonization during early life is critical for proper immune and physiological development in later life. Research has shown that colonization of the gut begins in humans when the fetus is in the lower uterus, but the gut microbiota develops and becomes established after birth ${ }^{[6]}$. Two main stages are involved in the evolution and establishment of the infants' gut microbiota. The first stage occurs during breast or formula feeding. After aerobic or facultative anaerobes, such as Eschericha coli and other Enterobacteriaceae, exhaust the oxygen supply in the intestinal tract of newborns during the first few days of life, the microbiome becomes dominated by Bifidobacterium. The second stage occurs after weaning of babies onto more diverse and solid foods, which results in a more complex microbiome, more similar to that of adults, whose microbiomes mainly contain Bacteroidetes and Firmicute ${ }^{[7][8]}$. The gut microbiota of the infant gradually evolves into that of an adult, gradually maturing and stabilizing at the age of 3 years into a microflora dominated by anaerobes and aerobes, a microecology maintained throughout youth and middle age ${ }^{[9]}$.

The establishment and development of the intestinal flora of infants are affected by a variety of factors. In previous studies, the contribution of delivery mode, feeding mode, and exposure to antibiotics and probiotics were examined ${ }^{[10][11][12]}$. However, environmental smoke exposure and duration of breastfeeding have not been evaluated. Smoking is common in China; in 2015, a total of $27.7 \%$ of the population smoked ${ }^{[13]}$. Because of cultural influences, more men smoke than women, but women are exposed passively to secondhand smoke and third-hand smoke (i.e., smoke that remains on environmental surfaces, such as clothes, hair, and dust $)^{[14]}$. Passive smoking, also known as environmental tobacco smoke exposure, is harmful to pregnant women and infants ${ }^{[15]}$. 
The father or grandparents will inevitably (during care taking) expose newborn babies to smoke and create a passive smoking environment. Studies have shown that passive smoking is harmful to the lungs and cardiovascular system, and sometimes passive smokers are more vulnerable to its harmful effects than active smokers ${ }^{[16]}$. Smokers have a lower diversity of gut bacteria than non-smokers, which leads to gut microbiota dysbiosis and can result in different diseases, such as inflammatory bowel diseases ${ }^{[17]}$. Smoking during pregnancy can lead to adverse pregnancy outcomes, such as preterm birth and low birthweight ${ }^{[18]}$, have lasting effects, such as neurodevelopmental abnormalities, behavioral problems, irritability, and attentional decline at 1 year of age $^{[19]}$. Children exposed to tobacco smoke are also more likely to have neurocognitive deficits, respiratory problems, and other diseases ${ }^{[20]}$. The relationship between intestinal flora and environmental tobacco exposure has been investigated in just a few studies in mice, and this research has shown that changes can occur in the bacterial composition and mucosal barrier function.

Previous studies have shown that breastfeeding is a major factor contributing to the colonization of infant guts. Infants fed breast milk have a higher abundance of Lactobacilli and Bifidobacteria than those fed formula milk ${ }^{[21][22]}$. A number of breast milk components, such as human milk oligosaccharides $(\mathrm{HMOs})^{[23]}$, promote colonization of beneficial bacteria, such as Bifidobacterium and Lactobacillus, and promote the absorption and digestion of nutrients ${ }^{[24]}$. The beneficial bacteria in breast milk are conducive to regulating the microecology of the intestinal flora, which can prevent imbalances in bacterial flora and increased drug resistance, both of which can be caused by antibiotic treatment ${ }^{[25]}$. Past studies have shown that, as a baby's diet transitions to mostly solid foods, their intestinal flora matures, becoming more complex and adult-like ${ }^{[26]}$, but another study suggests that the increase in complexity happens instead during weaning ${ }^{[22]}$. Research has shown that Bifidobacteria and Lactobacillus spp. are the dominant bacteria in baby intestines at the beginning of weaning, and that later, with the addition of supplementary food, the intestinal flora acquires a low abundance of Clostridium leptum, Clostridium coccoides, and Roseburia spp. The microbiota of the infant fed formula milk at the same stage contains a higher proportion 
of Bacteroides, Clostridium difficile, Clostridium perfringens, and Clostridium coccoides (a gut of a lower maturity) ${ }^{[27]}$. Although the effects of weaning on the microbiota of infants has been documented in some studies, a cohort follow-up study detailing the effects of weaning duration on microbiota composition in children aged 0-2 years has not been done.

The general dynamics of infant intestine colonization; the characteristics of the infant gut microflora as it develops toward an environment similar to that of the mother; and the influence of the delivery mode, antibiotics, and probiotics on infant flora have been well characterized. However, the characteristics of the intestines at different stages before the age of 3 years, when the microbiome stabilizes, has not been well elucidated. Also, the influence of environmental tobacco smoke exposure and weaning time on the development of the infant intestinal flora are largely understudied.

In this study, our objective was to (1) characterize the composition and evolution of the gut microbiota in 0-2-year-old children at different time points, (2) verify that the microbiota of young children becomes more similar to that of their mother as they get older, and (3) investigate the influence of environmental tobacco smoke exposure and weaning time on infant gut microbiota composition at different ages. Characterizing infant microbiota development and evaluating whether and how the environment and caretaker behaviors influence the bacterial composition of the gut will provide new perspectives and enable the promotion of healthy infant intestinal microbiota development. 


\section{Materials and Methods}

\subsection{Study type and design}

This study had a prospective cohort design. We recruited mothers being treated by the Department of Obstetrics and Gynecolog in Zhongnan Hospital, a hospital affiliated with Wuhan University (Wuhan, HuBei Province, China), to participate in our study. A total of 37 mother-child pairs were included and followed up for 2 years, and this whole project was conducted during March 4, 2017-July 1, 2019. This study was approved by the Research Ethics Boards of the medical school at Wuhan University (March 3, 2017, JKHL2017-03-03). All fecal samples and information were collected with the informed consent of participants.

Pregnant women included in this study were those who planned to give birth in Zhongnan Hospital and were Wuhan residents. We excluded women who smoked, had pregnancy complications, received antibiotics during pregnancy, or who had cognitive impairments.

\subsection{Variables and measures}

Fecal samples were collected from mothers in their third trimesters and from children at 6 months, 12 months, and 24 months of age. Mothers completed general questionnaires pertaining to themselves during their third trimester, after delivery, and at 12 months and completed questionnaires pertaining to their children at 6,12 , and 24 months after delivery. In the original cohort, we had 50 mother-child pairs; however, 13 pairs were lost to follow-up. We ended up including just the 37 mother-child pairs from whom we had complete sets of data and fecal samples. In addition, we used information from hospital records, the prenatal health manual, and the neonatal health manual.

The information collected about mothers included height, pre-pregnancy weight, late pregnancy weight, weight gain during pregnancy, infant gestational age at birth, delivery mode, drinking alcohol during pregnancy and lactation, and number of pets at home. Pre-pregnancy weight was classified as underweight, normal weight, overweight, or obese according to Asian-Pacific recommendations ${ }^{[28]}$. Using women's pre-pregnancy weight and gestational weight gain (GWG), we categorized mothers into the groups 
insufficient growth, normal growth, and excessive growth according to the classification criteria recommended by the Institute of Medicine ${ }^{[29]}$.

Information collected about children included secondhand environmental tobacco smoke exposure frequency (categorized as $0,1-2,3-4,5-6$, or 7 days/week), alcohol exposure during fetal development (yes or no), feeding by breast milk (yes or no) and duration of breastfeeding (usually within 0-12 months), feeding patterns at 6 and 12 months (breast milk + complementary food, breast milk + milk powder, milk powder + complementary food, and breast milk + milk powder + complementary food), length of weaning period (the point that weaning ended), and antibiotic and probiotic exposure (during 0-6 months, 7-12 months, and 13-24 months).

\subsection{Fecal sample collection}

The uniform fecal collection tube was used (centrifuge rube; $15 \mathrm{ml}$, Corning). All samples were collected at participants' homes by researchers which were trained professionally. During the collection, the uncontaminated feces inside were taken with cotton swabs and put into the tube. After recording the information such as coding and time, they were put into the transfer box containing dry ice, and then transferred to the refrigerator at $-80^{\circ} \mathrm{C}$ until ready for analysis. The whole process was less than 2 hours.

\subsection{Extraction and sequencing of genomic DNA from intestinal flora}

All 148 fecal samples were subjected to genomic DNA extraction and high-quality Illumina sequencing process in batches. We amplified the V3-V4 region of 16S rRNA using previously described primers (338F [5'-ACTCCTACGGGAGGCAGCAG-3'] and 806R [5'-GGACTACHVGGGTWTCTAAT-3'] $)^{[14]}$. The PCR solution contained $4 \mu \mathrm{L} 5$ $\times$ FastPfu buffer solution, $2 \mu \mathrm{L}$ dNTPs $(2.5 \mathrm{mmol} / \mathrm{L}), 0.8 \mu \mathrm{L}$ of each primer $(5 \mu \mathrm{mol} / \mathrm{L})$, $0.4 \mu \mathrm{L}$ FastPfu polymerase $(5 \mathrm{~mol} / \mathrm{L})$, and $10 \mathrm{ng}$ DNA template. The thermocycler program used was $95^{\circ} \mathrm{C}$ for 3 min (initial denaturation); 27 cycles of $95^{\circ} \mathrm{C}$ for $30 \mathrm{~s}$, $55^{\circ} \mathrm{C}$ for $30 \mathrm{~s}$ (annealing), and $72{ }^{\circ} \mathrm{C}$ for $45 \mathrm{~s}$ (elongation); and $72{ }^{\circ} \mathrm{C}$ for $10 \mathrm{~min}$ (final extension). PCR products were run on and purified from $2 \%$ agarose gels in accordance with sequencing requirements. Using purified PCR products, we constructed MiSeq libraries (Illumina, San Diego, CA, USA) and sequenced. We spliced together 16S RNA data and used Flash (https://ccb.jhu.edu/software/FLASH/index.shtml) for quality 
control; we then clustered sequences with high similarity $(>97 \%)$ into the smallest operational taxonomic units (OTUs) by Uparse (http://www.drive5.com/uparse/).

\subsection{Statistical methods}

We described general demographic information using percentages. To evaluate species quantity in or richness of fecal samples, pan/core species analyses were conducted The pan species analysis was used to indicate whether the total number of species increased with the volume of sample (i.e., whether the level of sequencing performed was sufficient). The fecal characteristics (species diversity [Shannon, Simpson], richness [sobs, chao, ace], and uniformity [heip, Shannoneven]) of each group of data was evaluated by Mothur (https://www.mothur.org/wiki/Download_mothur). We considered increased Shannon index values indicative of increased diversity.

A community composition analysis was conducted for each group of data. We obtained the taxonomic classifications (boundary, phylum, class, order, family, genus, species) of each species and presented the dominant species and relative abundances by using a community bar chart. To explore gut microflora differences in children of different age groups, the linear discriminant analysis effect size for each group was determined to find the species at different taxonomic classification levels (from phylum to genus) significantly different between groups. One-way analysis of variance was used to show trends in the variation of species and their relative abundance at the three different ages we examined, which represent different development stages.

To study the similarities and differences in bacterial communities in the four different sets of fecal samples, a principal coordinates analysis was conducted by using the unweighted UniFrac distance algorithm.

Because some environmental factors could have been strongly colinear, variance inflation factor (VIF) was used to screen out weak collinear environmental factors to eliminate their influence on subsequent correlation analyses. VIF is expressed as: $\mathrm{VIF}_{\mathrm{i}}=$ $1 /\left(1-\mathrm{R}_{\mathrm{i}}^{2}\right)$. Large VIF values indicate strong multicollinear relationships between independent variables. Environment factors with a VIF value $>10$ are generally 
considered to be extraneous. We filtered out environment factors with VIF values $>10$ and conducted multiple screenings until all VIF values for all selected environmental factors were $<10$.

A heatmap analysis was conducted to calculate the Spearman rank correlation coefficients between environmental factors and species of flora in children's intestines at different ages. The environmental factors evaluated were child sex, delivery mode, parity, GWG, mother's alcohol drinking behavior during gestation and lactation, having pets, child's feeding pattern, length of weaning period, exposure to tobacco smoke, number of family members, exposure of child to antibiotics and probiotics during the different stages (0-6, 7-12, and 13-24 months), child illness (diarrhea, respiratory disease, and fever) during the different stages (0-6, 7-12, and 13-24 months), and child weight and height at 24 months. In the heatmap analysis, we considered 0.4 (i.e., the absolute value) to be the threshold indicating significantce. If a value exceeded $(+$ or -$)$ 0.4 , a correlation existed, and the higher the value, the greater the correlation. 


\section{Results}

\subsection{Population characteristics}

A total of 37 mother-child pairs were included in the study. A stool sample was obtained from each mother during their third trimester and from children at 6,12 , and 24 months of age (totaling 148 stool samples). The average \pm SD number of days mothers were pregnant was $279 \pm 6.44$. The average \pm SD birth weight of neonates was $3.4 \pm 0.36 \mathrm{~kg}$, and average birth height was $50.5 \pm 1.34 \mathrm{~cm}$. Fifteen women had vaginal births and 22 had caesarean births (Table 1); 25 neonates were girls and 12 were boys (Table 2). The mean \pm SD weaning time of children was $12.5 \pm 6.02$ months. Other general information collected about mothers (GWG and alcohol consumption) and children (feeding pattern, exposure to environmental tobacco smoke, exposure to pets at home, and treatment with antibiotics and probiotics) are shown in Tables 1-3.

\subsection{Characteristics of sequencing results}

We obtained a total of 7,223,112 high-quality sequences from 37 mother-child pairs (using 148 samples) using Illumina MiSeq sequencing; the average number of sequences per sample was 48,804 . After data trimming and quality filtering to an equal sequencing depth, we classified sequences into 1212 OTUs and 14 phyla. The pan species analysis curve (Figure 1) showed that the number of OTUs increased with the number of samples, and the sequencing quantity was sufficient. Bacteria were classified by species, and 14 different phyla, 100 different families, and 273 different genera were detected from 111 children samples.

\subsection{The diversity of gut microbiota in each group}

Using 148 fecal samples, we compared gut microbiota diversity between mothers and children and by child's age $(6,12$, and 24 months). The coverage was $>99 \%$, and no statistical difference in coverage was found between groups (Supplemental table 1, inter-group $t$ test data about index), indicating that the sequencing depth was sufficient to cover most microorganisms, including rare species. The mean values of sobs, chao, and ace showed that the richness in intestinal flora of children increased with age. Likewise, the Shannon index (indicator of community diversity) also increased with age, 
and the diversity of the intestinal flora of the oldest children (24 months of age) was more similar to that of their mothers. The species richness and diversity and uniformity among samples from mothers was higher than those of their children at all time points.

\subsection{Composition and changes of microflora at different child ages}

We detected bacteria of 184 different genera at 6 months, 173 genera at 12 months, and 225 genera at 24 months. Genera with a relative abundance $<1 \%$ were combined. The relative abundance of 27 genera were $>1$ (Figure 2). The top 10 genera detected in 6-month-old infants were Bifidobacterium (38.45\%), Escherichia and Shigella (9.40\%), Clostridium sensu stricto cluster 1 (7.97\%), Veillonella (7.32\%), Bacteroides (6.33\%), Enterobacter (4.39\%), Ruminococcus gnavus group (3.98\%), Megasphaera (3.86\%), Enterococcus (1.91\%), and Streptococcus (1.77\%). The top 10 genera found in 12-month-old children were Veillonella (21.59\%), Bifidobacterium (19.21\%), Bacteroides (9.44\%), Escherichia and Shigella (8.47\%), Ruminococcus gnavus group (6.83\%), Clostridium sensu stricto cluster 1 (3.86\%), Blautia (3.71\%), Feacalibacterium (2.89\%), Megasphaera (2.72\%), and Lachnoclostridium (2.59\%). The top 10 genera in 24-month-old children were Bifidobacterium (29.20\%), Enterococcus (10.18\%), Blautia (8.07\%), Escherichia and Shigella (7.42\%), Lactobacillus (4.83\%), Ruminococcus gnavus group (3.51\%), Streptococcus (2.62\%), Bacteroides (2.18\%), Eubacterium hallii group (2.03\%), and Subdoligranulum (1.67\%).

The relative abundances of Bifidobacterium, Veillonella, and Enterococcus were significantly different among 6-month-old, 12-month-old, and 24-month-old children (P $\leqslant 0.001)$. Bifidobacterium and Enterococcus decreased in abundance in the guts of children 12 months of age and then appeared increased in the sample acquired at 24 months. The relative abundance of Bifidobacterium and Enterococcus was highest at 6 months, and the relative abundance of Enterococcus was highest at 24 months. Veillonella increased in abundance from the 6-month time point to the 12-month time point and then was decreased in the fecal samples acquired at 24 months. Other flora, such as Bacteroides, Blautia, and Clostridium sensu stricto cluster 1 all changed significantly during early childhood development (Figure 3). 


\subsection{Similarity between the intestinal floras of children and mothers}

The top three bacteria (in relative abundance) in the mother's intestinal tract were Bacteriodes (20.79\%), Faecalibacterium (13.16\%), and Prevotella (11.56\%) (Figure 2). Bifidobacterium, the predominant bacterium in fecal samples of children at each time points, constituted only $1.72 \%$ of the mother's intestinal flora. The richness and diversity of the mother's intestinal flora were higher than those of the children's flora at all ages examined, and the richness and diversity of the children's flora increased with time, approaching the maturity of the mother's (Supplemental table 1). The similarity of the intestinal flora among the four groups was studied by principal coordinate analysis. Of the 3 age groups, the children 24 months of age had microflora most similar to that of their mothers, and the microflora of infants 6 months of age was least similar (Figure $4)$.

\subsection{Factors influencing differences in intestinal flora in infants}

VIF analysis was conducted for all environmental factors at each time point to screen out environmental factors with multicollinear relationships. Environmental factors with VIF values $>10$ were filtered out. Supplemental table 2 shows the VIF values of environmental factors before and after screening. The VIF values of all environmental factors were $<10$ (i.e., they had weak collinearity), and no influence on subsequent correlation analyses were found.

Heatmap analyses showed the presence of a correlation between environmental factors and child gut microbiota at different time points (Figure 5). A Spearman correlation analysis was used to calculate the threshold value (absolute value of 0.4 ). Figure 5A shows correlations between gut microbiota and various factors at 6 months of age. Weaning time was positively correlated with the abundance of Lactobacillus $(\mathrm{R}=0.4815 ; 0.001<\mathrm{P} \leqslant 0.01)$, and GWG was positively correlated with the abundance of Flavonifractor $(\mathrm{R}=0.4923 ; 0.001<\mathrm{P} \leqslant 0.01)$; the more exposure a baby had to secondhand and third-hand smoke during the breastfeeding stage of development, the lower the abundance of Flavonifractor in their intestinal flora $(\mathrm{R}=0.4583 ; 0.001<\mathrm{P} \leqslant$ $0.01)$. 
Figure 5B shows the factors affecting microbiota compositon of infants 12 months of age. At this age, weaning was still positively correlated with the abundance of Lactobacillus $(\mathrm{R}=0.4658 ; 0.001<\mathrm{P} \leqslant 0.01)$, but the association was a little decreased compared with that found at 6 months. Environmental tobacco smoke exposure was not correlated with the abundance of Flavonifractor but did correlate with the Blautia abundance $(\mathrm{R}=0.4061 ; 0.01<\mathrm{P} \leqslant 0.05)$. At this time point, antibiotic exposure also correlated with Blautia abundance, showing that treating children with antibiotics can decrease the abundance of this bacterium in their intestines $(\mathrm{R}=-0.4295 ; 0.001<\mathrm{P} \leqslant$ 0.01). For the fecal samples acquired during June-December, the incidence of respiratory diseases (general colds, influenza, and pneumonia), diarrhea, and fever was evaluated. During this period, respiratory diseases were correlated with the abundance of Lactobacillus $(\mathrm{R}=0.4026 ; 0.01<\mathrm{P} \leqslant 0.05)$.

\subsection{Effect of environmental tobacco smoke exposure on gut microbiota of young children 6-24 months of age}

We analyzed the diversity of the microbiota (Shannon index) of children exposed and not exposed to environmental tobacco smoke at 6, 12, and 24 months (6 groups) and used the Welch $t$ test to detect the intergroup differences (Figure 6, Supplemental table 3). At 6 months of age, the Shannon index differed between the children exposed to environmental tobacco smoke and those not $(\mathrm{P}=0.03362)$. No significant differences were found between exposed and non-exposed children at 12 and 24 months of age. We used linear discriminant analysis effect size to analyze the differences in abundance of species (from phylum to genus) in exposed and non-exposed children at 6 months of age (Figure 7). The abundance of the phylum Firmicutes ( family Ruminococcaceae) was elevated in children not exposed to environmental tobacco smoke, and the abundance of Actinobacillus (family Pasteurellaceae) was higher in children who were. 


\section{Discussion}

\subsection{The diversity of intestinal flora of young children increased with age and gradually approached the maturity of their mothers.}

This study showed that Firmicutes and Actinobacteria predominated the intestinal flora of children before the age of 2 years, and Firmicutes and Bacteroidetes predominated the mother's flora. Early studies showed that $>90 \%$ of the adult bacterial flora is composed of Firmicutes, Bacteroidetes, and Actinomycetes ${ }^{[4]}$, and in later studies, two genera, Bacteroides and Prevotella, were found to constitute the majority ${ }^{[30]}$. Consistent with previous studies, the predominant three genera of bacteria in pregnant women in this study were Bacteroidetes, Faecobacterium, and Prevotella ${ }^{[31]}$. At birth, babies guts are characterized predominantly by Enterococcus and Staphylococcus ${ }^{[32]}$. In this study, we see that with the addition of breast milk to babies' diets, flora becomes mainly characterized by Bifidobacterium and Lactobacillus, and this intestinal flora composition is consistent until babies are fed solid foods. After the weaning period, the gut microflora of children tends to mature, with increased species diversity, mainly including Bacteroidetes, Prevotella, and Ruminococcus ${ }^{[33]}$. In our study, Bifidobacterium was the predominant genus present at all three time points during early childhood development, and Ruminococcus made up a large proportion at 6 months and 12 months but decreased significantly at 24 months. Compared with the 6-month time point, at 12 months and 24 months, the abundance of Ruminococcus was increased, indicating that the intestinal flora was gradually maturing. These microorganisms produce short-chain fatty acids and effectively degrade dietary fiber, enhancing the function of carbohydrates, and the presence of Ruminococcus typically indicates that the child's intestinal environment has transformed into that similar to that of an adult ${ }^{[8]}$. In our study, the average weaning time was around 12 months, and we found that longer weaning times promoted the maturation of the baby's microflora. Therefore, during the period from 12 months to 24 months, most children had completed weaning and began to eat a diet similar to that of an adult, so their intestinal microflora at 12 months and 24 months was more adult-like. 
The diversity of microbiota of children's intestines increased gradually from 6 months to 24 months, with the greatest variation seen among children 24 months of age. This finding is similar to those found in a previous study showing higher diversity among 2-year-olds but smaller differences among children 24 months of age ${ }^{[31]}$. There are many factors that influence the diversity of intestinal flora. In this study, alcohol and antibiotic exposure and disease status were all associated with specific flora but had no effect at 6 and 12 months. Antibiotic use can lead to imbalances in intestinal flora and reduced diversity ${ }^{[34]}$, and diarrhea is associated with the abundance of Blautia ${ }^{[35]}$. In our study, treatment with antibiotics, infectious diseases, and diet appeared to cause the greatest differences in microbiota in children aged 24 months.

Of the three age groups evaluated in this study, the children 24 months of age had the intestinal flora most similar to that of their mothers. The results of a Swedish study showed that the microbiota of 1-year-old infants was more similar to that of their mothers than to the microbiota of 4-month-old infants, a finding consistent with the trend we observed of gradual maturation of intestinal microbiota with age (from birth to 3 years $)^{[8]}$.

\subsection{Weaning time completion was associated with Lactobacillus abundance, with longer weaning times associated with higher Lactobacillus abundance.}

Breast milk is rich in HMOs, and $>200$ HMOs have been found. These oligosaccharides are indigestible but play a vital role in helping develop the gut microbiome and building a young immune system ${ }^{[36]}$. Because HMOs are difficult to digest, they pass through the stomach and small intestine and eventually accumulate in the colon ${ }^{[37]}$. One of the functions of the HMO lactose is to facilitate the colonization of Bifidobacterium and (similar to probiotics) prevent the colonization of pathogenic bacteria. In addition to HMOs, breast milk also contains probiotics that promote the development of intestinal flora. Studies have shown that breast-fed infants consume up to 800,000 bacteria per day and when breastfeeding ceases the fecal flora of infants begins to be similar to that of their mothers ${ }^{[27]}$. The source of the bacteria in breast milk is unclear, but studies have shown that the bacteria probably originates from a 
combination of locations (the baby's mouth and the mother's nipples and skin) $)^{[38]}$. Therefore, breastfeeding extensively facilitates the colonization of beneficial bacteria and prevents colonization of harmful bacteria in the intestinal flora of infants. In this study, the length of weaning was associated with the abundance of Lactobacillus; however, feeding patterns showed no correlations with specific bacteria genera at any of the three time points. A 3-year cohort study about infants' microbiota in Danish showed that especially during 9 to 18 months, compared with infants who stopped breastfeeding, breastfeeding for a longer period of time was associated with a higher abundance of Bifidobacterium, Lactobacillus, and Veillonella ${ }^{[39]}$, a finding consistent with our study (that a higher abundance of Lactobacillus was associated with longer weaning times).

Studies have shown that Lactobacillus reuteri can effectively reduce the crying times of some infants but also has a regulatory effect on the microflora of infants receiving antibiotics ${ }^{[40]}$; this bacterium appeared to eliminate the changes in microbial diversity and drug resistance typically induced by antibiotics. In addition, experiments in pigs have shown that Lactobacillus added to lactoid foods increased the concentration of short-chain fatty acids in intestines and promotes the secretion of glucagon-like peptide-1 ${ }^{[41]}$, thus regulating the function of pancreas islets. Providing Lactobacillus rhamnosus $G G$-containing foods to infants at high risk for asthma, who often experience delayed intestinal development, resulted in improvements in their gut regulatory environment ${ }^{[42]}$. Therefore, Lactobacillus plays an important role in the regulation of infant gut flora; the prevention of diarrhea ${ }^{[43]}$, asthma, and other diseases; and the promotion of the healthy infant growth. Besides Lactobacillus, other bacteria in breast milk, such as Bifidobacterium ${ }^{[44]}$, also participate in maintaining intestinal homeostasis. The World Health Organization recommends exclusive breastfeeding of infants up to the age of 6 months ${ }^{[45]}$. The longer weaning occurs, the higher the abundance of beneficial bacteria in the intestinal flora of infants, and this type of environment is highly conducive to the heathy development of infant neuroimmunity. 


\subsection{Environmental tobacco smoke exposure decreased the diversity of bacteria in 6-month-old infants and was associated with the abundance of Blautia in 12-month-old infants. The more exposure, the lower the abundance of Blautia.}

Studies have shown that carbon monoxide and nicotine are the two main toxins in cigarettes. Carbon monoxide has a stronger affinity than oxygen to combine with hemoglobin, which leads to a decrease in blood oxygen and blood flow and affecting the development of the brain. Nicotine can cross the blood-brain barrier and has an effect on a variety of neurotransmitter activities, such as receiving pleasure through the release of dopamine and influencing cognition through serotonin, norepinephrine, and aminobutyric acid ${ }^{[46]}$.

The gastrointestinal tract plays a role in immune function. In recent years, investigators have paid more attention to this topic, and the interaction between the intestinal flora and brain central nervous system has become a research focus ${ }^{[47]}$. Existing research shows that gut bacteria and the brain can affect each other via the gut-brain axis through mechanisms involving the neural, endocrine, and immune pathways. Cigarette smoke exposure affects brain dysplasia (potentially through the influence of a diverse intestinal microflora increasing the production of inflammatory factors); thus, the neuroendocrine pathways might alter the development of the brain ${ }^{[48]}$. In an animal study, nicotine was shown to interfere with the transmission of information between the brain and nerves of the gut by affecting neurotransmitters, such as glutamic acid, aminobutyric acid, and uric acid; this interference resulted in disregulated brain-to-gut biochemical signaling and an unbalanced intestinal microecosystem ${ }^{[49]}$.

Another study in mice showed that fluid-based smoking led to an increase in Clostridium spp. and a decrease in Firmicutes (mainly Lactobacillus and Ruminococcus spp. $)^{[50]}$. In human studies, active smokers had a higher proportion of Clostridium difficile in their intestinal flora than those who had quit smoking before the study began or those who had never smoked ${ }^{[51]}$. A study of smoking adults showed a higher abundance of Erysipelotrichia, Catenibacterium, and Alphaproteobacteria in smokers than nonsmokers ${ }^{[52]}$. In our study, 6-month-old infants exposed to cigarette smoke had a 
higher diversity in gut bacteria than 6-month-old infants not exposed to cigarette smoke. Unlike the findings of studies conducted with adults, our results did not show an increase in Alphaproteobacteria in infants who were exposed to smoke, potentially because the infant gut microbiota is developing and different (less complex) than that of adults. However, the abundance of Blautia decreased with the increase in smoke exposure frequency at 6 months and 12 months. This result might have been due to the effect of nicotine on intermediate neurotransmitters of the gut-brain axis. Blautia plays a role in reducing inflammation and balancing intestinal microecology ${ }^{[35]}$. Therefore, environmental tobacco exposure negatively affects intestinal flora homeostasis, and long-term exposure might also cause cognitive development defects in infants through mechanisms involving the gut-brain axis. Therefore, further research determining the exposure levels the affect the gut-brain axis is needed.

\section{Limitations}

There are still some limitations to this study that should be considered when interpreting results. First, no quantitative analysis of environmental tobacco smoke exposure was performed. We relied on family members' self-reporting children's exposures, and they might have misremembered or intentionally underreported exposures out of guilt. Second, we did not collect samples from newborns and cannot explain the colonization from the very begining. Third, we did not collect fecal samples at the beginning and completion of weaning to compare the differences in microflora composition before and after weaning. Fourth, our sample size was small and should be increased in future studies exploring the evolution of the gut microbiota with age. Last, we followed children for only 2 years, when the microflora of children is generally considered to be stable at 3 years. In future studies, follow-up should be extended to 3 years to explore the dynamic changes occurring in the microflora of young children. Despite these limitations, this study contributes to the literature because it is the first to explore whether environmental tobacco smoke exposure influences the composition of the intestinal flora of children at different ages. 


\section{Conclusion}

The gut microbiomes of young children become more diverse (and more like that of their mothers) with age. The maturation of microflora is influenced by the length of time infants spend weaning. In addition, exposure to environmental tobacco smoke is associated with an imbalanced microflora in infants, and the greater the exposure to tobacco, the lower the abundance of Blautia in their microflora. According to the results of this study, breastfeeding for $>6-12$ months can promote the production of beneficial bacteria in infant intestines, supporting the World Health Organization breastfeeding recommendations. Also, reducing infant exposures to tobacco smoke should improve children's health by preventing dysbiosis of their gut microbiota.

\section{References}

[1] Gill SR, Pop M, Deboy RT, et al. Metagenomic analysis of the human distal gut microbiome. Science. 2006;312(5778):1355-1359

${ }^{[2]}$ Gut microbiome dysbiosis and increased intestinal permeability in children with islet autoimmunity and type 1 diabetes: A prospective cohort study[J]. Pediatric Diabetes, 2019, 20(5).

[3] Axelsson P B , Clausen T D , Petersen A H , et al. Relation Between Infant Microbiota and Autism?: Results from a National Cohort Sibling Design Study[J]. Epidemiology, 2018, 30(1):1.

[4] Mbakwa Catherine A,Hermes Gerben D A,Penders John,Savelkoul Paul H M,Thijs Carel,Dagnelie Pieter C,Mommers Monique,Zoetendal Erwin G,Smidt Hauke,Arts Ilja C W. Gut Microbiota and Body Weight in School-Aged Children: The KOALA Birth Cohort Study.[J]. Obesity (Silver Spring, Md.),2018,26(11).

${ }^{[5]}$ Durack J , Kimes N E, Lin D L, et al. Delayed gut microbiota development in high-risk for asthma infants is temporarily modifiable by Lactobacillus supplementation[J]. Nature Communications, 2018, 9(1):707.

[6] Tanaka M , Nakayama J . Development of the gut microbiota in infancy and its impact on health in later life[J]. Allergology International, 2017, 66(4):515-522. 


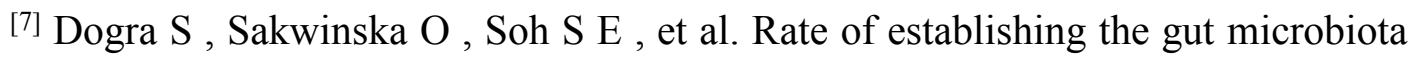
in infancy has consequences for future health[J]. Gut Microbes, 2015:1-5.

${ }^{[8]}$ Dogra S, Sakwinska O, Soh S E, et al. Dynamics of Infant Gut Microbiota Are Influenced by Delivery Mode and Gestational Duration and Are Associated with Subsequent Adiposity[J]. mBio, 2015, 6(1):e02419-14.

${ }^{[9]}$ Contreras M , Magris M , Hidalgo G, et al. Human gut microbiome viewed across age and geography[J]. Nature, 2012, 486(7402):222-227.

${ }^{[10]}$ Zhang, D.X., et al., Effects of Different Modes of Delivery and Feeding on Intestinal Flora of Newborns and Infants with Different Ages. Iranian Journal of Pediatrics, 2019. 29(4).

${ }^{[11]}$ Haejin, Kim, Alexandra, et al. Birth Mode, Breastfeeding, Pet Exposure, and Antibiotic Use: Associations With the Gut Microbiome and Sensitization in Children.[J]. Current allergy and asthma reports, 2019. doi:10.1007/s11882-019-0851-9

${ }^{[12]}$ Rutayisire E , Huang K , Liu Y, et al. The mode of delivery affects the diversity and colonization pattern of the gut microbiota during the first year of infants $\ "$ life: a systematic review[J]. BMC Gastroenterology, 2016, 16(1):86.

[13] Mark G , Rong Z . Tobacco control and Healthy China 2030[J]. Tobacco Control, 2018:tobaccocontrol-2018-054372-.

[14] Protano C , Vitali M. The New Danger of Thirdhand Smoke: Why Passive Smoking Does Not Stop at Secondhand Smoke[J]. Environmental Health Perspectives, 2011, 119(10):a422-a422.

${ }^{[15]}$ Xia X , Yan L, Xiaoxiao S, et al. Discrepancy between Self-Reported and Urine Cotinine-Verified Environmental Tobacco Smoke Exposure among Rural Pregnant Women in China[J]. International Journal of Environmental Research and Public Health, 2018, 15(7):1499-.

${ }^{[16]}$ Rui-Mei F , Shang-Ying H , Fang-Hui Z , et al. Role of active and passive smoking in high-risk human papillomavirus infection and cervical intraepithelial neoplasia grade 2 or worse[J]. Journal of Gynecologic Oncology, 2017, 28(5):e47-. 


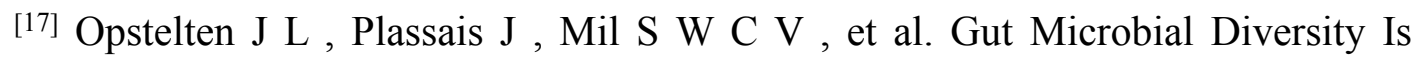
Reduced in Smokers with Crohn's Disease[J]. Inflammatory Bowel Diseases, 2016, 22(9):2070-2077.

${ }^{[18]}$ McLean Cara,Jun Shelly,Kozyrskyj Anita. Impact of maternal smoking on the infant gut microbiota and its association with child overweight: a scoping review.[J]. World journal of pediatrics : WJP,2019,15(4).

${ }^{[19]}$ Zhou S , Rosenthal D G, Sherman S, et al. Physical, Behavioral, and Cognitive Effects of Prenatal Tobacco and Postnatal Secondhand Smoke Exposure[J]. Current Problems in Pediatric and Adolescent Health Care, 2014, 44(8):219-241.

${ }^{[20]}$ Ekblad M , Korkeila J , Lehtonen L . Smoking during pregnancy affects foetal brain development[J]. Acta Paediatrica, 2015, 104(1):12-18.

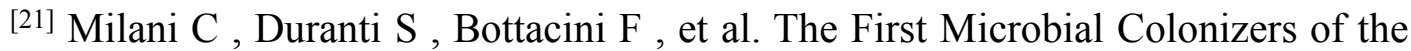
Human Gut: Composition, Activities, and Health Implications of the Infant Gut Microbiota[J]. Microbiology and Molecular Biology Reviews, 2017, 81(4):e00036-17.

${ }^{[22]}$ Backhed F , Roswall J , Peng Y, et al. Dynamics and Stabilization of the Human Gut Microbiome during the First Year of Life[J]. Cell Host \& Microbe, 2015, 17(5):690-703.

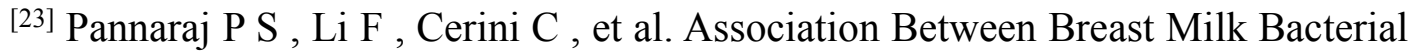
Communities and Establishment and Development of the Infant Gut Microbiome[J]. Jama Pediatr, 2017, 171(7):647-654.

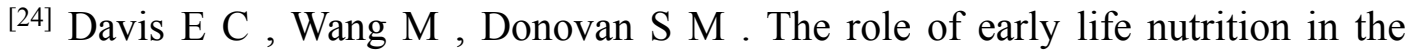
establishment of gastrointestinal microbial composition and function[J]. Gut Microbes, $2017,8(2): 143-171$.

${ }^{[25]} \mathrm{Li}$, Y.H., et al., Oral administration of Lactobacillus delbrueckii during the suckling period improves intestinal integrity after weaning in piglets. Journal of Functional Foods, 2019. 63.

[26] Laursen M F , Bahl M I , Michaelsen K F , et al. First Foods and Gut Microbes[J]. Frontiers in Microbiology, 2017, 8.

${ }^{[27]}$ Moore Rebecca E,Townsend Steven D. Temporal development of the infant gut microbiome.[J]. Open biology,2019,9(9) 
${ }^{[28]}$ Pan W H , Yeh W T . How to Define Obesity? Evidence-based Multiple Action Points for Public Awareness, Screening, and Treatment: an Extension of Asian-Pacific Recommendations[J]. Asia Pacific Journal of Clinical Nutrition, 2008, 17(3):370-374.

${ }^{[29]}$ Medicine I O . Weight Gain During Pregnancy: Reexamining the Guidelines[J]. National Academies Press, 2009. doi:http://dx.doi.org/

${ }^{[30]}$ Knights D , Ward T L , Mckinlay C E , et al. Rethinking “Enterotypes”[J]. Cell Host \& Microbe, 2014, 16(4):433-437.

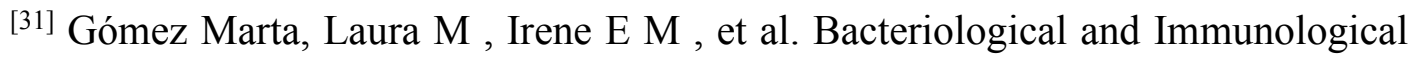
Profiling of Meconium and Fecal Samples from Preterm Infants: A Two-Year Follow-Up Study[J]. Nutrients, 2017, 9(12):1293-. doi:10.3390/nu9121293

${ }^{[32]}$ Zhang W , Li J , Lu S, et al. Gut microbiota community characteristics and disease-related microorganism pattern in a population of healthy Chinese people[J]. Scientific Reports, 2019, 9(1). doi:10.1038/s41598-018-36318-y

${ }^{[33]}$ Yvonne Vallès, Artacho A , Alberto Pascual-García, et al. Microbial Succession in the Gut: Directional Trends of Taxonomic and Functional Change in a Birth Cohort of Spanish Infants[J]. PLoS Genetics, 2014, 10(6):e1004406.

[34] Scott H. Podolsky. Historical Perspective on the Rise and Fall and Rise of Antibiotics and Human Weight Gain[J]. Annals of Internal Medicine, 2017, 166(2):133.

[35] Wong M L , Inserra A , Lewis M D , et al. Inflammasome signaling affects anxiety- and depressive-like behavior and gut microbiome composition[J]. Molecular Psychiatry, 2016. doi:10.1038/mp.2016.46

[36] Tamburini S, Shen N, Wu H C, et al. The microbiome in early life: implications for health outcomes[J]. Nature Medicine, 2016, 22(7):713-722.

[37] Human breast milk: A review on its composition and bioactivity[J]. Early Human Development, 2015, 91(11):S0378378215001772.

${ }^{[38]}$ Kirsty L D , Beth H , Aisha B , et al. Mother' s Milk: A Purposeful Contribution to the Development of the Infant Microbiota and Immunity[J]. Frontiers in Immunology, 2018, 9:361-. doi:10.3389/fimmu.2018.00361 
[39] Bergstrom A, Skov T H , Bahl M I , et al. Establishment of Intestinal Microbiota during Early Life: a Longitudinal, Explorative Study of a Large Cohort of Danish Infants[J]. Applied and Environmental Microbiology, 2014, 80(9):2889-2900.

${ }^{[40]}$ Nation M L , Dunne E M , Joseph S J , et al. Impact of Lactobacillus reuteri colonization on gut microbiota, inflammation, and crying time in infant colic[J]. Scientific Reports, 2017, 7(1):15047.

${ }^{[41]}$ Marion L , Samir D , Armelle C , et al. Addition of dairy lipids and probiotic Lactobacillus fermentum in infant formula programs gut microbiota and entero-insular axis in adult minipigs[J]. Scientific Reports, 2018, 8(1):11656-. doi:10.1038/s41598-018-29971-w

${ }^{[42]}$ Durack J , Kimes N E , Lin D L, et al. Delayed gut microbiota development in high-risk for asthma infants is temporarily modifiable by Lactobacillus supplementation[J]. Nature Communications, 2018, 9(1):707.

[43] Yinghui Li,Shuling Hou,Jiashun Chen,Wei Peng,Wei Wen,Fengming Chen,Xingguo Huang. Oral administration of Lactobacillus delbrueckii during the suckling period improves intestinal integrity after weaning in piglets[J]. Journal of Functional Foods,2019,63.

[44] Effects of Probiotic Supplementation on the Gut Microbiota and Antibiotic Resistome Development in Preterm Infants.[J]. Frontiers in pediatrics, 2018. doi:10.3389/fped.2018.00347

$[45]$

$$
\text { WHO. Undated. }
$$

Complementary

feeding.

See https://www.who.int/nutrition/topics/complementary_feeding/en/.

[46] Froggatt Suzanne,Covey Judith,Reissland Nadja. Infant neurobehavioural consequences of prenatal cigarette exposure: A systematic review and meta-analysis.[J]. Acta paediatrica (Oslo, Norway : 1992),2019. doi:10.1111/apa.15132

[47] Osadchiy Vadim,Martin Clair R,Mayer Emeran A. Gut Microbiome and Modulation of CNS Function.[J]. Comprehensive Physiology,2019,10(1).

${ }^{[48]}$ Fricker Michael,Goggins Bridie J,Mateer Sean,Jones Bernadette,Kim Richard Y,Gellatly Shaan L,Jarnicki Andrew G,Powell Nicholas,Oliver Brian G,Radford-Smith Graham,Talley Nicholas J,Walker Marjorie M,Keely Simon,Hansbro Philip M. Chronic 
cigarette smoke exposure induces systemic hypoxia that drives intestinal dysfunction.[J]. JCI insight,2018,3(3).

[49] Chi Liang,Mahbub Ridwan,Gao Bei,Bian Xiaoming,Tu Pengcheng, Ru Hongyu,Lu Kun. Nicotine Alters the Gut Microbiome and Metabolites of Gut-Brain Interactions in a Sex-Specific Manner.[J]. Chemical research in toxicology,2017,30(12).

${ }^{[50]}$ Allais Liesbeth,Kerckhof Frederiek-Maarten,Verschuere Stephanie,Bracke Ken R,De Smet Rebecca,Laukens Debby,Van den Abbeele Pieter,De Vos Martine,Boon Nico,Brusselle Guy G,Cuvelier Claude A,Van de Wiele Tom. Chronic cigarette smoke exposure induces microbial and inflammatory shifts and mucin changes in the murine gut.[J]. Environmental microbiology,2016,18(5).

${ }^{[51]}$ Rogers M A M , Greene M T , Saint S , et al. Higher Rates of Clostridium difficile Infection among Smokers[J]. PLOS ONE, 2012, 7.

[52] Nolan-Kenney Rachel,Wu Fen,Hu Jiyuan,Yang Liying,Kelly Dervla,Li Huilin,Jasmine Farzana,Kibriya Muhammad G,Parvez Faruque,Shaheen Ishrat,Sarwar Golam,Ahmed Alauddin,Eunus Mahbub,Islam Tariqul,Pei Zhiheng,Ahsan Habibul,Chen Yu. The association between smoking and gut microbiome in Bangladesh.[J]. Nicotine \& tobacco research : official journal of the Society for Research on Nicotine and Tobacco,2019. doi:10.1093/ntr/ntz220

\section{Figure legend}

Figure 1. Pan species analysis

Figure 2. Composition of gut flora of mothers and children at 6, 12, and 24 months of age.

Figure 3. Change in composition of gut microbiota of children from 6 to 24 months of age; $\mathrm{P}$ value, $* 0.01<\mathrm{P} \leqslant 0.05, * * 0.001<\mathrm{P} \leqslant 0.01, * * * \mathrm{P} \leqslant 0.001$

Figure 4. Scatterplot from PCoA of genera present in fecal samples acquired from mothers during their third trimester and children at 3 different ages. Analysis was based on unweighted UniFrac distance of the MetaOTUs in each sample. OUT, operational taxonomic unit; PCoA, principle coordinate analysis. 
Figure 5. Heatmap of Spearman correlation analysis comparing environmental factors with the genera of flora detected in infants at 6 months (A) and 12 months (B) of age. The $\mathrm{R}$ value of the correlation is indicated by color, with blue as negative and red as positive Values greater than the absolute value of 0.4 were considered significant. $* 0.01$ $<\mathrm{P} \leqslant 0.05, * * 0.001<\mathrm{P} \leqslant 0.01, * * * \mathrm{P} \leqslant 0.001$.

Figure 6. Differences in $\alpha$ diversity of gut microbiota of children 6 months of age, 12 months of age, and 24 months of age exposed and not exposed to environmental tobacco smoke. Diversity was measured by determining the Shannon index at the OTU level, and differences were assessed by using the Welch $\mathrm{t}$ test. Medians and interquartile ranges are indicated in boxes; whiskers indicate ranges. OTU, operational taxonomic unit. $* 0.01<\mathrm{P} \leq 0.05 ; * * 0.001<\mathrm{P} \leq 0.01 ; * * * \mathrm{P} \leq 0.001$.

Figure 7. Differences in gut microbiota species (from phylum to genus) between 6-month-old infants exposed (blue) and not exposed (red) to environmental tobacco smoke depicted by cladogram (A) and LEfSe bar graph (B). Differences were measured by using LEfSe. The LDA score obtained by LDA analysis (linear regression analysis), the larger the LDA score, the greater the impact of species abundance on the differential effect. LEfSe, linear discriminant analysis effect size.

\section{Acknowledgements}

Thanks to Shanghai Majorbio Bio-pharm Technology Co.,Ltd for its technical support in sample sequencing.

\section{Supplemental tables}

Supplemental table $1 \underline{\text { a diversity index table.xls }}$

Supplemental table 2 Environmental screening table.xlsx

Supplemental table 3 Exposure vs. Non-exposure in group 6,12,24 months 


\section{Availability of data and materials}

The data were analyzed on the free online platform of Majorbio Cloud Platform (www.majorbio.com). Raw data of the MiSeq sequencing can be provided if needed.

The data mentioned in the article are listed in tables (including supplemental tables) and figures.

\section{Funding}

This work was funded by Natural Science Foundation of Hubei Province ( grant number 2017CFB343) and the Key Independent Scientific Research Project of WuHan University School of Health Sciences ( grant number JSZD2016001).

\section{Author information}

\section{Affiliations}

Tianqu Xie (2015302280014@whu.edu.cn), Jin He(jing26@whu.edu.cn), Yun Yu(yuyun7169@163.com), Yuchen Wang(wang-yuchen@whu.edu.cn), Yanqu Liu and Xiaoli Chen : Affiliation Wuhan University School of Health Sciences, Wuhan University, 169 Donghu Road, Wuhan 430071, China;

Jinbing Bai(jinbing.bai@emory.edu): Affiliation Emory University Nell Hodgson Woodruff School of Nursing, 1520 Clifton Road, Atlanta, GA 30322, USA;

Contributions

J.H, Y.Y and Y.W collected the fecal data and other information.

T.X analysed the data and wrote the manuscript.

J.B reviewed and edited this article.

Y.L supervised the whole project and reviewed and edited the article.

X.C supervised the project.

Corresponding authors

Correspondence to Yanqun Liu (Tel: 027-68758591, E-mail: 1iuyanqun1984@163.com) or Xiaoli Chen (Tel: 027-68758591, E-mail: chenx172@whu.edu.cn).

\section{Ethics declarations}

This study was approved by the Research Ethics Boards of the medical school at Wuhan University (March 3, 2017, JKHL2017-03-03). 


\section{Consent to publish}

All fecal samples and information were collected with the informed consent of participants.

\section{Competing interests}

No competing interests. 
Figures

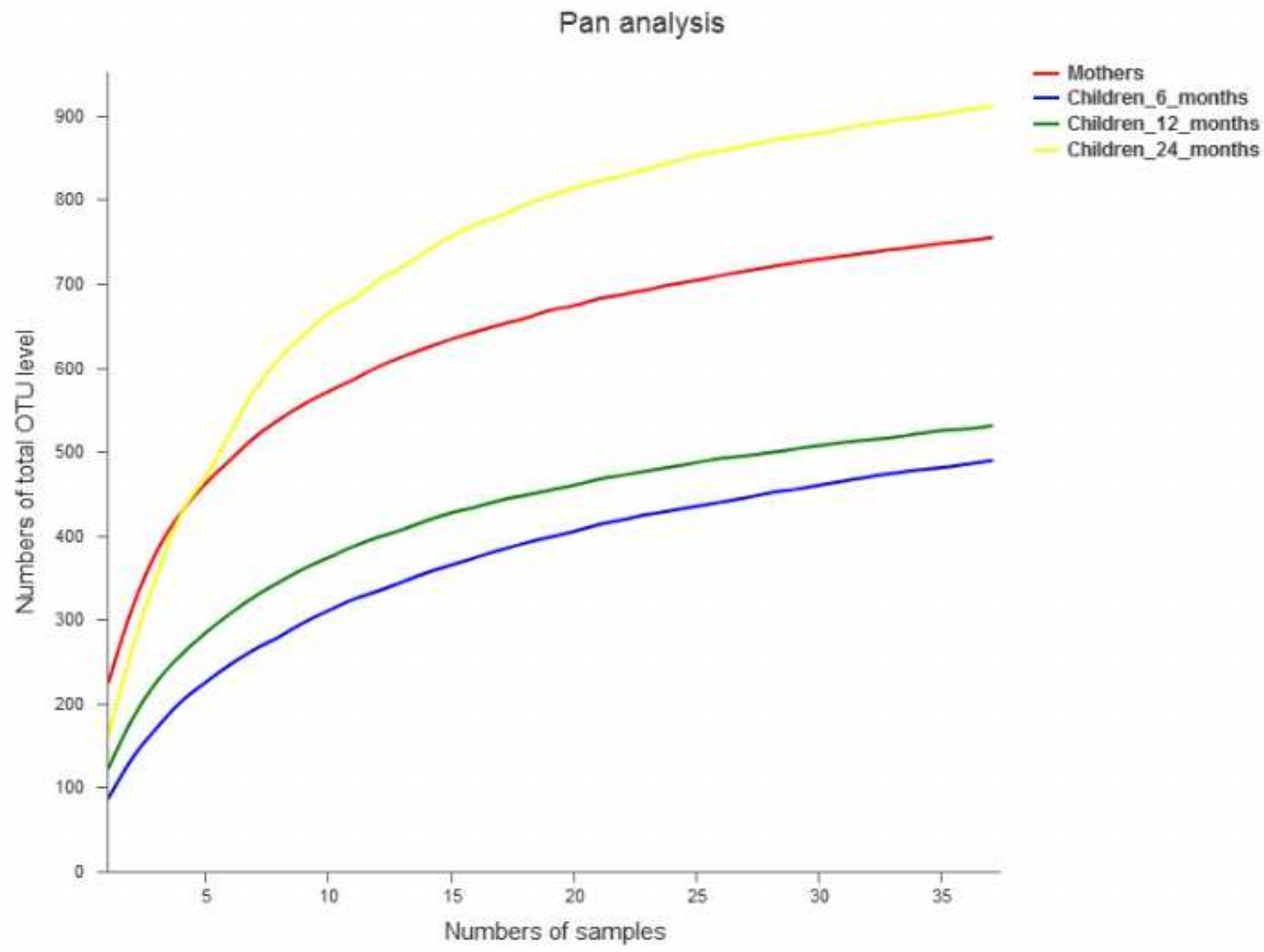

Figure 1

Pan species analysis 


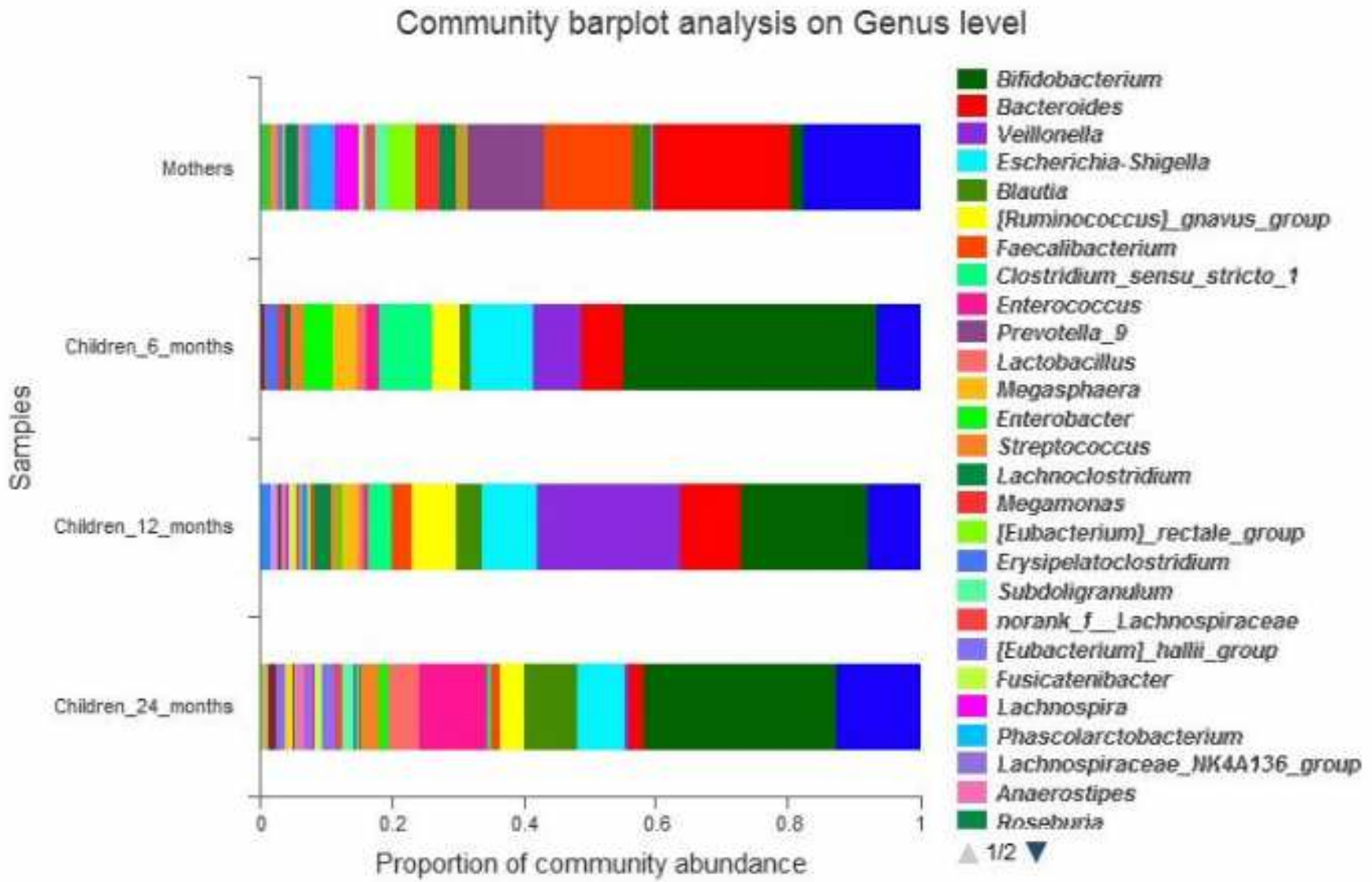

Figure 2

Composition of gut flora of mothers and children at 6,12 , and 24 months of age. 


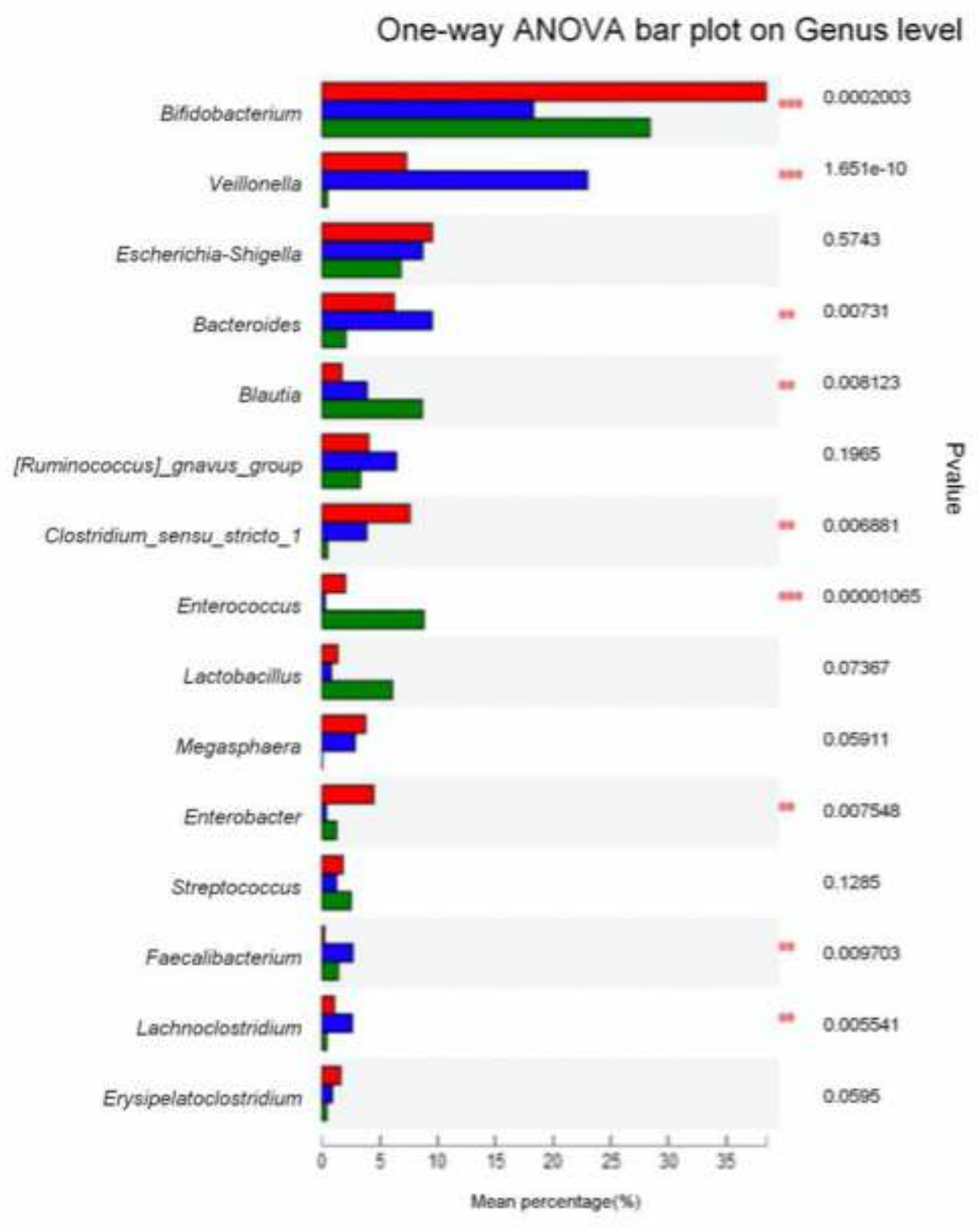

Figure 3

Change in composition of gut microbiota of children from 6 to 24 months of age; $\mathrm{P}$ value, $* 0.01<\mathrm{P} \leq$

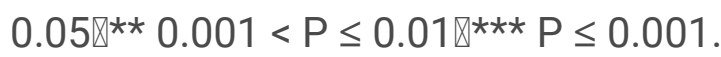




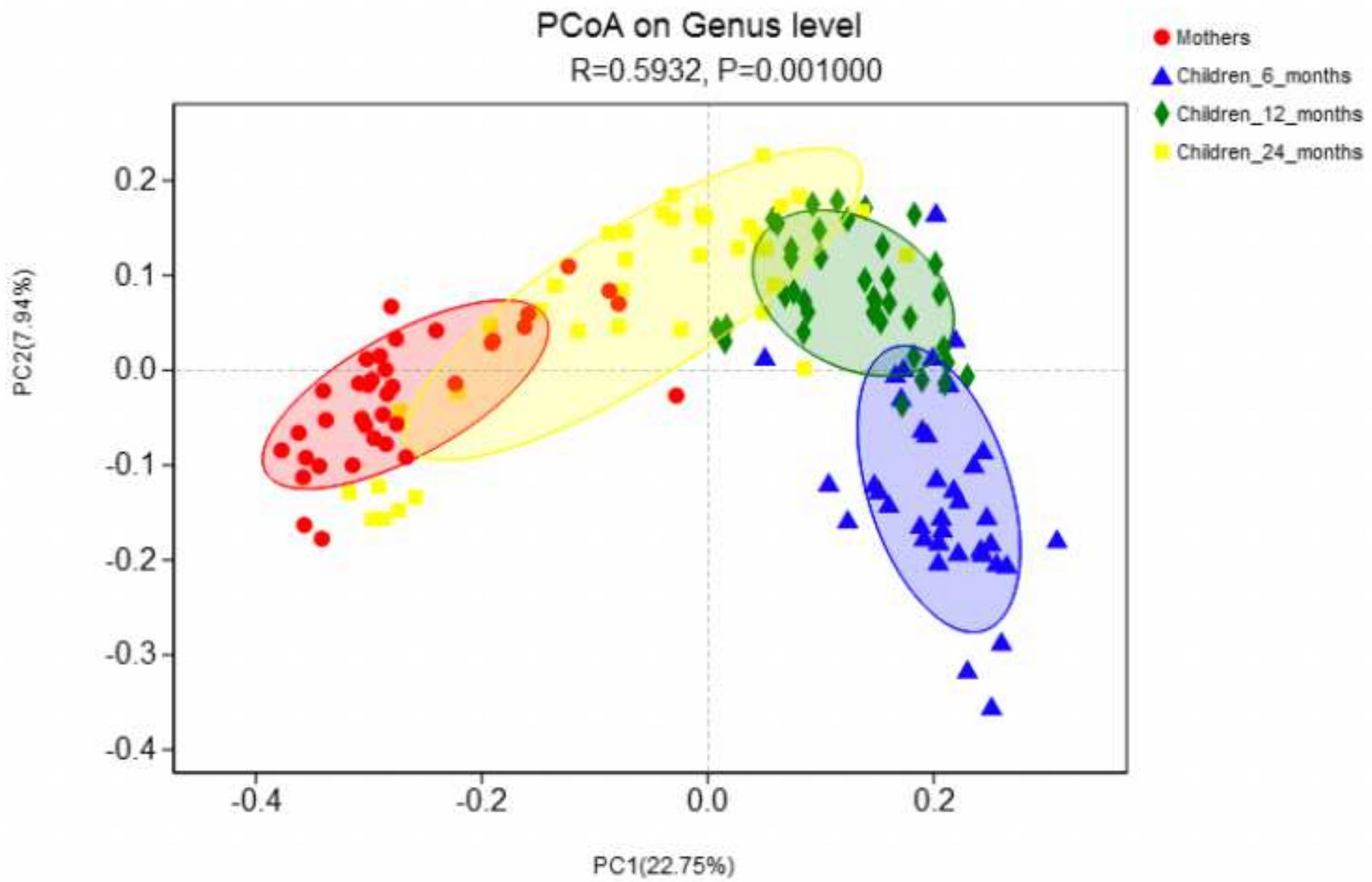

Figure 4

Scatterplot from PCoA of genera present in fecal samples acquired from mothers during their third trimester and children at 3 different ages. Analysis was based on unweighted UniFrac distance of the MetaOTUs in each sample. OUT, operational taxonomic unit; PCoA, principle coordinate analysis. 


\section{Spearman correlation of 6-month-old children on Genus}

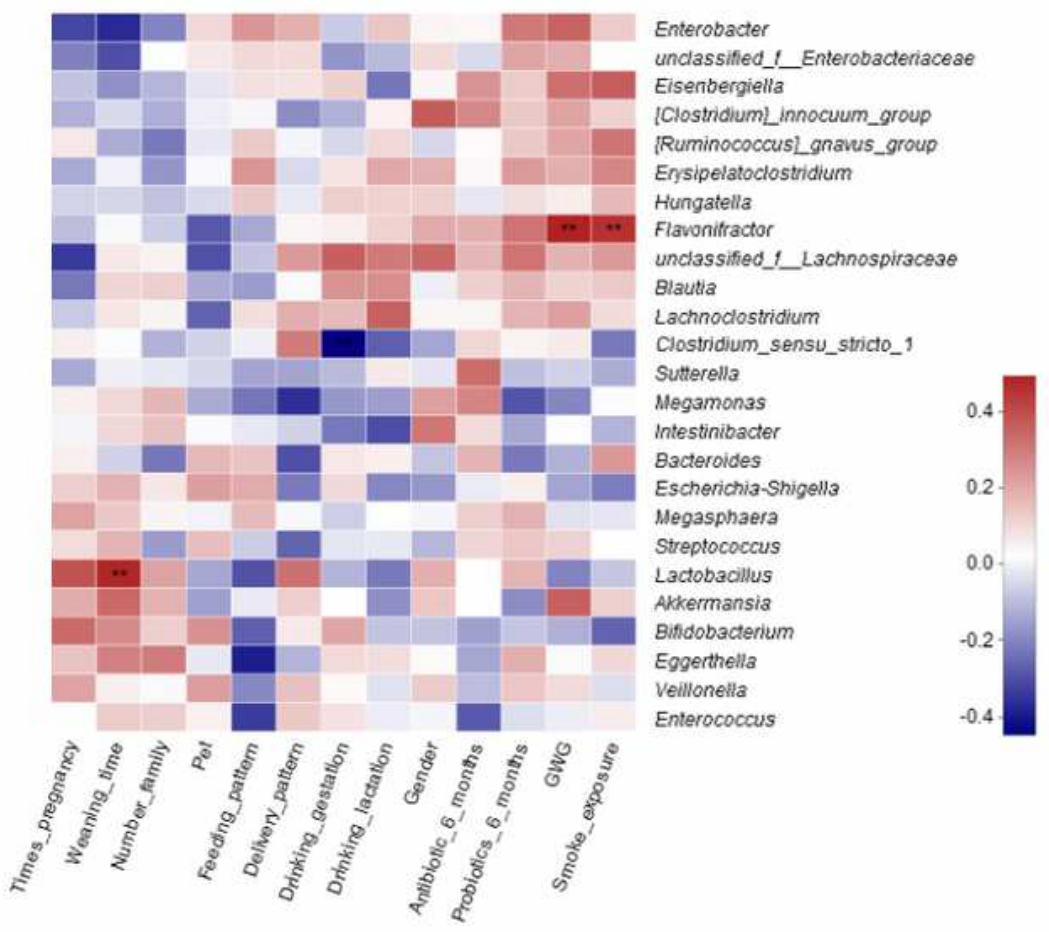

\section{Spearman correlation of 12-month-old children on Genus}

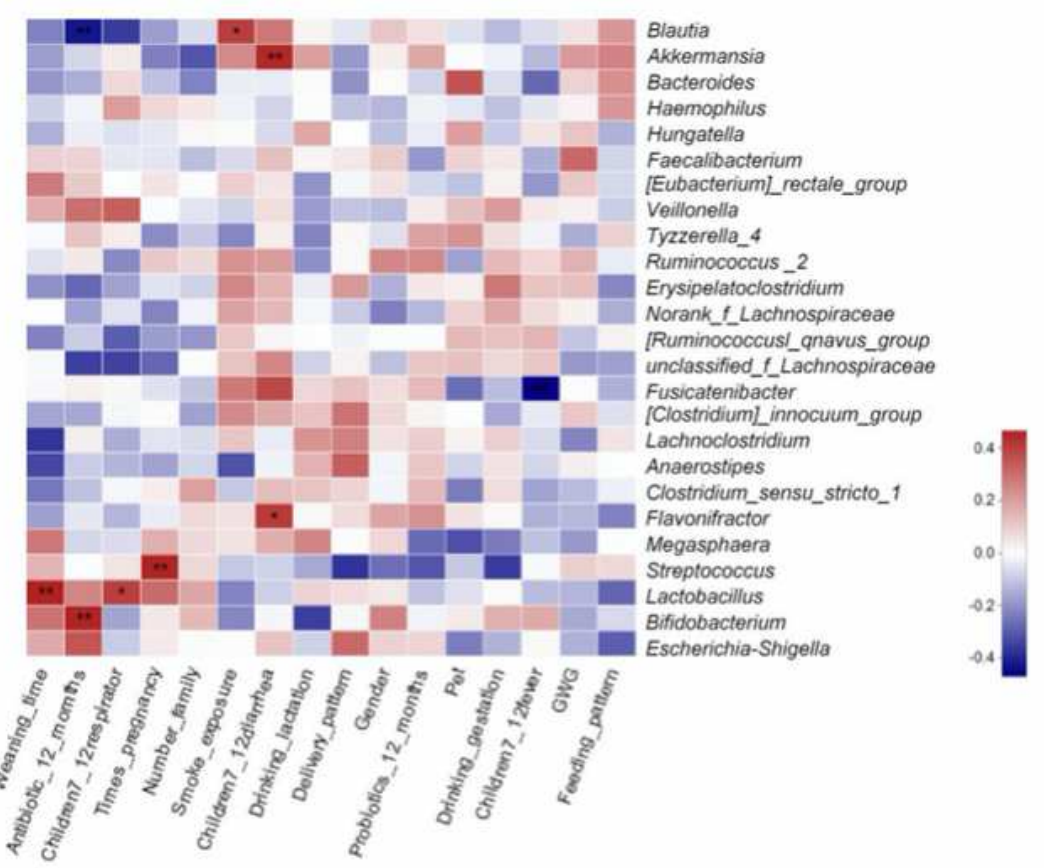

\section{Figure 5}

Heatmap of Spearman correlation analysis comparing environmental factors with the genera of flora detected in infants at 6 months (A) and 12 months (B) of age. The $R$ value of the correlation is indicated by color, with blue as negative and red as positive Values greater than the absolute value of 0.4 were considered significant. * $0.01<P \leq 0.05 \rrbracket^{\star \star} 0.001<P \leq 0.01 \rrbracket^{\star \star \star \star} P \leq 0.001$. 


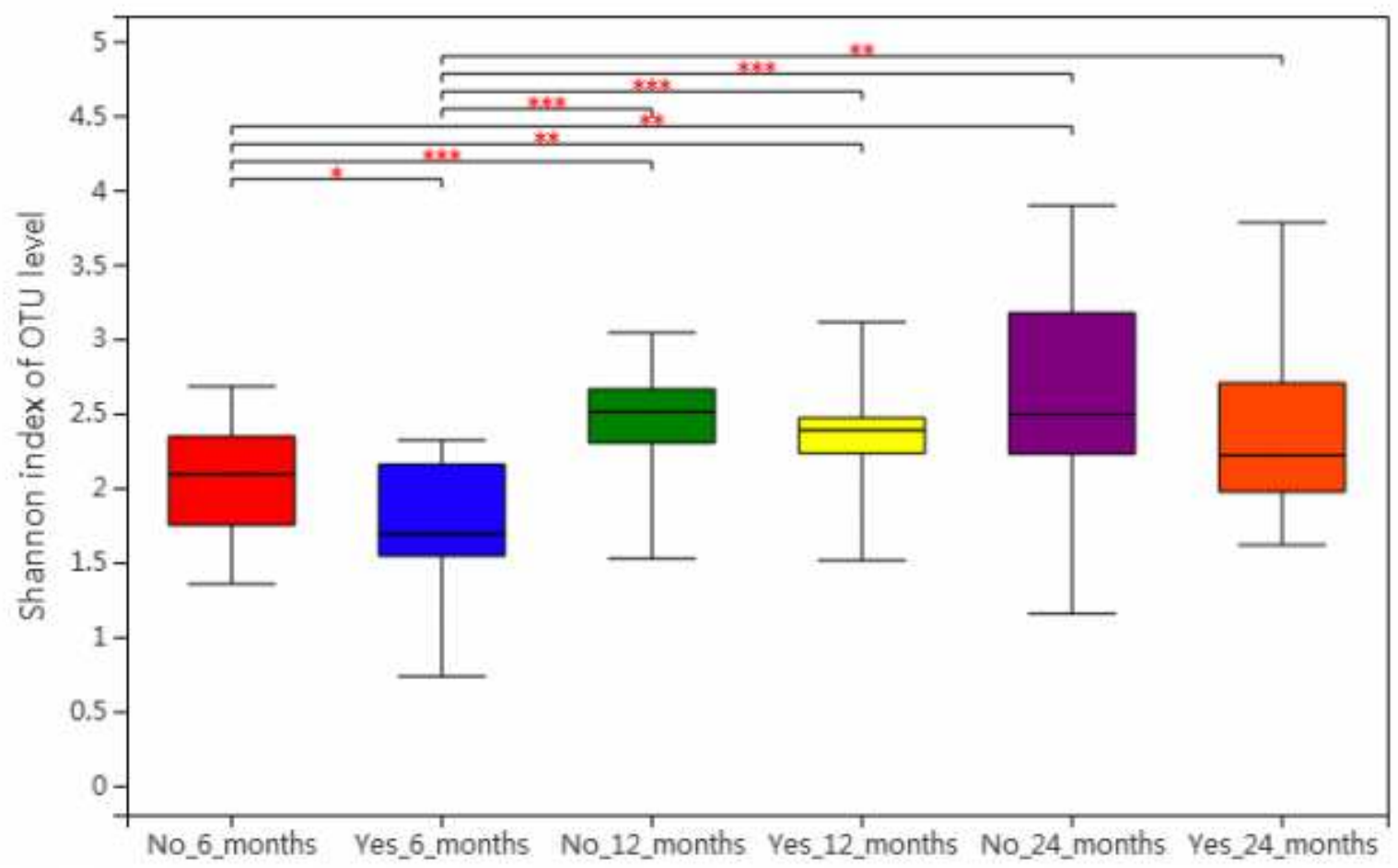

No_6_months

Yes_6_months

No_12_months

Yes_12_months

No_24_months

Yes_24_months

\section{Figure 6}

Differences in a diversity of gut microbiota of children 6 months of age, 12 months of age, and 24 months of age exposed and not exposed to environmental tobacco smoke. Diversity was measured by determining the Shannon index at the OTU level, and differences were assessed by using the Welch $\mathrm{t}$ test. Medians and interquartile ranges are indicated in boxes; whiskers indicate ranges. OTU, operational taxonomic unit. ${ }^{*} 0.01<\mathrm{P} \leq 0.05 ;{ }^{*} 0.001<\mathrm{P} \leq 0.01 ; * \star * \mathrm{P} \leq 0.001$. 

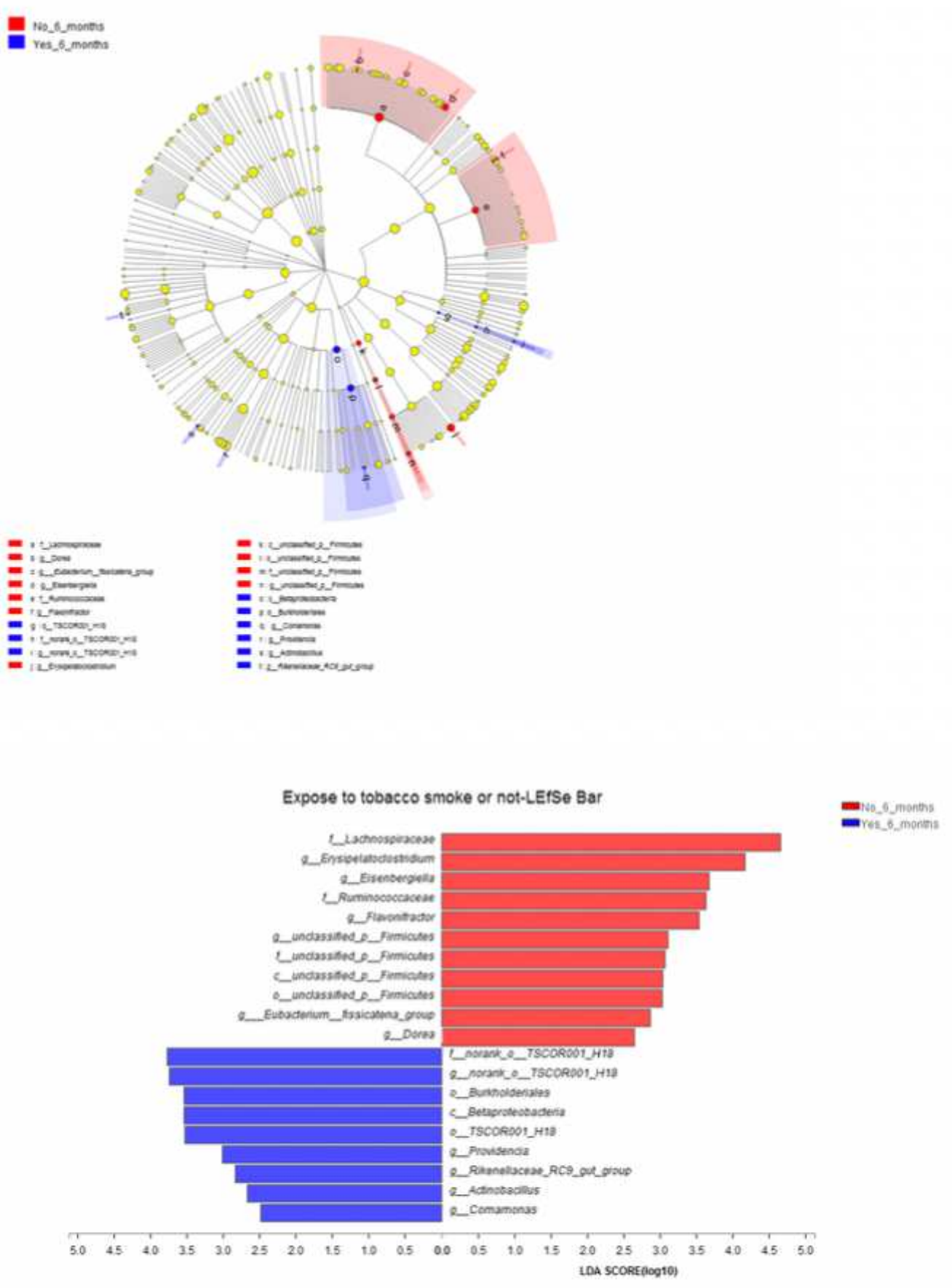

\section{Figure 7}

Differences in gut microbiota species (from phylum to genus) between 6-month-old infants exposed (blue) and not exposed (red) to environmental tobacco smoke depicted by cladogram (A) and LEfSe bar graph (B). Differences were measured by using LEfSe. The LDA score obtained by LDA analysis (linear regression analysis), the larger the LDA score, the greater the impact of species abundance on the differential effect. LEfSe, linear discriminant analysis effect size. 


\section{Supplementary Files}

This is a list of supplementary files associated with this preprint. Click to download.

- Environmentalscreeningtable.xlsx

- renamedb217b.xls 